Cahiers de Narratologie

Analyse et théorie narratives

$10.1 \mid 2001$

La voix narrative

\title{
Voix du narrateur et identification du lecteur
}

\section{Béatrice Bloch}

\section{(2) OpenEdition}

\section{Journals}

Electronic version

URL: http://journals.openedition.org/narratologie/6944

DOI: 10.4000/narratologie.6944

ISSN: 1765-307X

\section{Publisher}

LIRCES

\section{Printed version}

Date of publication: 1 January 2001

Number of pages: 221-229

ISBN: 2914561032

ISSN: 0993-8516

\section{Electronic reference}

Béatrice Bloch, "Voix du narrateur et identification du lecteur", Cahiers de Narratologie [Online], 10.1 I 2001, Online since 24 October 2014, connection on 23 February 2021. URL: http://

journals.openedition.org/narratologie/6944 ; DOI: https://doi.org/10.4000/narratologie.6944 


\title{
VOIX DU NARRATEUR ET IDENTIFICATION DU LECTEUR
}

\author{
Béatrice BLOCH \\ Université de Nice-Sophia Antipolis
}

Il reste une voix à l'œuvre, dans la littérature de la deuxième moitié du vingtième siècle, si abstraite celle-ci soitelle, si détachée, comme la parole peu lyrique de L'Étranger. On continue à lire pourtant. C'est que la lecture peut investir, de manière non-identificatoire, les images et les sons inscrits dans le texte' ${ }^{1}$. Mais est-ce tout?

Beaucoup de narrations récentes choisissent l'homodiégèse, la voix d'un narrateur se mettant en scène à la première personne ${ }^{2}$. Or, dans le cas d'un texte écrit à la première personne, où la voix s'entend d'autant plus qu'elle feint la communication orale avec son lecteur, le récit ne charrie-t-il pas inéluctablement une identification minimale du lecteur au narrateur-personnage? Cette voix du «je » qui s'écrit permet-elle au lecteur de partager une vision et une expérience avec l'énonciateur, d'établir une connivence avec un sujet, même fragmentaire, clivé, désarticulé ? Ou bien, au contraire, suffit-il, pour s'approcher du texte, de cette voix, usant de la première personne, sans qu'il soit besoin d'évoquer quelque système identificatoire que ce soit, sans que soit nécessaire le retour du sujet, disparu depuis Barthes et Foucault?

${ }^{1}$ Béatrice BLOCH, "Modernités farouches : Claude Simon, Kateb Yacine ", p. 150-162, in La Lecture littéraire, ${ }^{\circ} 3$, revue de Recherche sur la Lecture des Textes Littéraires (Université de Reims), Klincksieck, 1999.

2 Dominique RABATÉ souligne dans Vers une littérature de l'épuisement (José Corti, 1991) le paradoxe d'une littérature actuelle qui repose sur la voix affirmée d'un narrateur à la première personne, alors même que le sujet se fait évanescent à la manière d'un personnage de Beckett. 
Bien des textes récents se présentent sous forme de récits à la première personne, forme énonciative commune puisqu'elle est celle de la communication orale 3 . En même temps que ce choix de la narration homodiégétique adoptant l'allure d'une confidence orale, ces textes peuvent sembler difficiles à saisir car ils mettent en scène des « je » étranges, parfois fantomatiques. Or, l'utilisation du « je » par tous, sa circulation dans le dialogue, montre que la langue permet une contextualisation des embrayeurs qui dépende non seulement des conditions de l'énonciation, mais aussi de celles de la réception, laquelle, à l'écrit, concerne le lecteur. Je poserai donc une double question aux textes contemporains écrits à la première personne. D'abord, est-ce que le lecteur s'actualise dans le « je » qu'il lit comme s'il était l'énonciateur (le « je ») ou comme s'il était le destinataire (le «tu»)? Et ensuite, est-ce que, par la voix narrative à la première personne, le lecteur s'identifie à une simple voix ou bien, au contraire, à d'autres aspects de ce que représente un sujet dans une expérience ordinaire de dialogue (celui qui parle, celui qui a un point de vue, perçoit le monde par son propre corps, a des affects, pense et agit) ?

Pour répondre à ces questions, je relèverai les raisons expliquant que toute lecture d'une narration homodiégétique est actualisée par le lecteur en je ou en $t u$. En même temps, je me demanderai si le lecteur, partageant la voix du narrateur en actualisant le je, n'en vient pas à partager son point de vue. Mon hypothèse est qu'il persiste toujours une identification à un je sentant ou à un je percevant, même dans les textes présentant des je réduits à une présence fantomatique.

\section{Identification du lecteur à un $t u$}

La première raison d'une actualisation de l'énonciation par le lecteur est d'ordre linguistique : elle tient à la nature de toute communication en première personne. Benveniste a bien montré que si je est celui qui parle (ici, le narrateur), alors celui à qui le $j e$ s'adresse est un $t u$. Une telle définition de

3 Qu'on pense aux textes de Robert Pinget, à ceux de Claude Simon, de Louis-René des Forêts, de Beckett dans certains textes comme Comment c'est, de passages de textes de François Bon... 
l'usage de l'embrayeur de la première personne nous oriente vers l'idée qu'un texte recourant à la voix du je induit chez le lecteur une attitude d'identification au destinataire : il deviendrait le $t u$ de cette communication qui feint l'oral.

Dans le prolongement de ces constats, et les transposant dans le domaine littéraire cette fois, Käte Hamburger a montré, dans Logique des genres littéraires ${ }^{4}$, les spécificités des genres et de leurs systèmes énonciatifs : pour elle, tandis qu'un texte homodiégétique relève de la feintise, du fairesemblant, car il imite une communication orale naturelle, au contraire un récit hétérodiégétique relève de la fiction, c'està-dire de conventions de langage spécifiques aux récits et qui s'éloignent de l'usage du langage ordinaire. En effet, elle a constaté que l'utilisation de la troisième personne dans un récit, combinée à une focalisation interne (comme dans «il s'est senti tout joyeux à cette idée »), est une convention de narration car il est patent que, dans une situation orale, il est impossible à un sujet de parler d'un tiers, tout en ayant connaissance de la vie intérieure de ce dernier (la phrase «il s'est senti tout joyeux à cette idée » n'imite aucune assertion réelle, à la différence de « je me suis senti tout joyeux à cette idée ", qui est courante). De tels types de raisonnements induisent l'idée que tout énoncé à la première personne efface le caractère conventionnel de la narration : le discours se donne comme un dialogue oral, sans que le cadre de la fiction ne soit souligné par une mise en scène entraînant un usage spécifique du langage. L'énoncé en première personne est une mimésis du langage ordinaire. Or, selon Jean-Marie Schaeffer, dans Pourquoi la fiction, tout dispositif fictionnel comprend deux temps: d'abord un leurre (qui permette de s'immerger dans le dispositif fictif, comme s'il était réel) et d'autre part, une neutralisation de ce leurre, qui empêche, par exemple, d'être effrayé par le film de l'arrivée d'un train en gare de La Ciotat, comme le furent les premiers spectateurs de cinéma ${ }^{5}$. Nous déduirons de ces remarques que la narration à

${ }^{4}$ Käte HAMBURGER, Logique des genres littéraires, Paris, Seuil, 1986 (éd. orig. Stuttgart, 1977).

5 Jean-Marie SCHAEFFER, dans Pourquoi la fiction, Seuil, 1999, écrit, p. 189: "La situation d'immersion fictionnelle se caractérise 
la troisième personne accroît la neutralisation du leurre fictif - et donc la distance et la méfiance du lecteur - du fait de son caractère conventionnel. En revanche, si l'énonciation est à la première personne ( $D$ Demain, je vais au cinéma»), l'immersion dans le dispositif fictionnel est d'autant plus forte que le caractère conventionnel du discours est moins apparent. Dans ce cas, le lecteur se conçoit comme proche de la situation de conversation naturelle et neutralise le caractère fictif de la situation, ce qui lui permet de s'imaginer comme l'interlocuteur de l'énonciateur à la première personne. Le lecteur devient un $t u$.

Mais, allant plus loin, notre hypothèse est que le lecteur s'identifie parfois au je du narrateur, et, en tout cas, au moins au je percevant.

\section{Identification du lecteur au $\mathbf{j e}$ de la narration}

Le lecteur s'identifie à un je percevant (c'est-à-dire ayant une perception interne de son corps, mais aussi voyant, entendant, etc.) presque immédiatement, car, étant lui-même en situation de perception de signes écrits, il s'assimile aisément à un je du narrateur/personnage présenté en situation de perception. Christian Metz, pour le domaine du cinéma, a souligné dans Le Signifiant imaginaire le phénomène de projection du spectateur qui s'établit irrépressiblement : dans tout film, le spectateur s'identifie à l'instance voyante $^{6}$. Nous faisons ici l'hypothèse que de semblables phénomènes ont lieu encore plus dans la lecture d'un texte à la première personne (ou la vision d'un film en caméra subjective). Le lecteur s'identifie au corps percevant qu'il est devenu. Pour faire cette hypothèse, nous nous sommes inspirée librement des travaux de François Récanati autour de

donc par l'existence conjointe de leurres mimétiques préattentionnels et d'une neutralisation concomitante de ces leurres par un blocage de leurs effets au niveau de l'attention consciente".

${ }^{6}$ Christian METZ, dans Le signifiant imaginaire, écrit p. 119: «Il ne s'agit pas ici de l'identification du spectateur aux personnages du film (elle est déjà secondaire), mais de son identification préalable à l'instance voyante (invisible) qu'est le film lui-même comme discours, comme instance qui met en avant l'histoire et qui la donne à voir ». C'est d'autant plus le cas si l'instance voyante est aussi un sujet. 
la question de ce qui différencie l'embrayeur $j e$ des autres déictiques? ${ }^{7}$.

François Récanati montre que tandis que les déictiques (comme les déictiques spatiotemporels et les démonstratifs) sont définis par la situation d'énonciation de celui qui les émet et permettent un repérage spatial qui prend pour origine de référence celui qui parle, en revanche l'embrayeur je n'est pas défini par la situation d'énonciation. En effet, personne ne définit je en se disant: "voyons, qui est je ? Qui parle? C'est moi qui parle, donc c'est moi qui suis $j e »$. François Récanati, s'appuyant sur une hypothèse de Wittgenstein, dit que toute énonciation du mot je, si arbitraire l'expression soit-elle du fait du caractère conventionnel de la langue, est mise en rapport intuitivement par le sujet avec la perception immédiate de soi-même, sans que soit nécessaire une réflexion. En effet, dans le cas des autres déictiques que je, comme par exemple dans l'expression "regarde ce bateau », ces déictiques sont définis, non par perception interne, mais par la perception qu'a l'énonciateur de la situation spatiotemporelle où il se trouve. Or, cette situation peut donner lieu à des aberrations de perceptions (si l'on dit " regarde ce bateau », on peut croire qu'il s'agit d'un bateau, alors qu'en réalité il s'agit d'un reflet de bateau dans une glace, si l'énonciateur n'a pas bien conscience de sa situation physique d'être devant une glace et non devant une fenêtre). En revanche dans le cas de l'embrayeur je, l'énonciateur a une perception intuitive et proprioceptive de son corps qui fait qu'il n 'a pas besoin de vision, de perception externe de la situation pour savoir qui est $j e$ (nul besoin de regarder ses jambes pour savoir qu'elles sont croisées). Aussi, François Récanati conclut-il en disant que l'expression arbitraire et culturelle qu'est $j e$ est immédiatement liée à la perception intuitive que chacun a de soi-même, le je n'étant pas défini en

${ }^{7}$ François RÉCANATI, «Contenu sémantique et contenu cognitif des énoncés ", in Introduction aux sciences cognitives, Folio, Essais, 1992, p. 239 à 271. Et, pour plus de précisions, François RECANATI, Direct Reference : From Language to Thought, Oxford: Blackwell, 1993 (les chapitres 4 et 5, p. 63 à 94 , traitent de cette question du pronom $j e$ ). 
fonction d'un contexte spatiotemporel perçu par les sens, mais perçu directement de l'intérieur.

Revenons à la question de la lecture. Si écrire $j e$, c'est actualiser une certaine forme de présence à soi qui provoque un rapport immédiat à la perception de son propre corps, pouvons-nous en déduire que la lecture du je provoque chez le lecteur semblable perception?

Tout dépend de la phrase considérée. Nous pouvons dire que le lecteur a affaire à une communication mimant l'oral, s'il lit les phrases « je suis content » ou « je suis triste ». Au contraire, s'il lit une phrase comme « je suis debout» ou «j'ai les jambes croisées », cette phrase ne peut être prononcée dans le cadre d'une communication orale directe, car il serait inutile de donner une telle information à l'interlocuteur, puisque celui-ci pourrait voir de ses yeux la situation corporelle de l'énonciateur. Dans le cas d'une phrase comme « je suis assis ", la situation d'énonciation ne mime plus la communication naturelle et le lecteur est donc encore moins un auditeur. Il se projette désormais dans le je lu comme s'il s'agissait de son propre courant de conscience, certes représenté arbitrairement (car nous ne nous disons jamais « je suis assis » puisque nous le savons intuitivement), mais dans un courant de conscience qui traduirait, par la convention de la narration, le type d'information que chacun possède intuitivement sur soi-même, indépendamment de la situation d'interlocution. Nous pensons que de tels énoncés sont une représentation conventionnelle qui s'approcherait le plus possible d'une mimésis des informations intérieures qui, d'ordinaire, ne sont pas verbalisées par le sujet, mais connues intuitivement par lui grâce à la proprioception . $^{8}$

Si l'on veut résumer cela, on obtient les formules suivantes :

*Je+présent+état interne psychique (ex. «Je suis content»), alors le lecteur est en situation de dialogue imité : il se conçoit comme un $t u$

${ }^{8}$ De tels modes d'assertions correspondraient peut-être à ce que Dorrit Cohn appelle «psycho-récit » ou à ce que Genette, dans Figures $I I I$, trouvait problématique à rendre, de manière mimétique, par la narration: "le récit de pensées », cf. Figures III, p. 189 sq. 
*Je+présent+état corporel visible (ex. «Je suis assis »), alors le lecteur s'identifie au $j e$, parce qu'il s'agit d'une imitation des informations intérieures disponibles à chacun par proprioception (et non par situation d'interlocution).

Afin d'illustrer notre hypothèse, nous nous référerons à un texte de François Bon tiré de Prison. Décrivant un des prisonniers dans le chapitre intitulé Isolement, le narrateur le désigne d'abord à la troisième personne : "Le bruit. Il est devant la fenêtre ouverte, comme c'est le premier étage il s'est accroupi. Mais il ne fait pas comme les autres, d'avoir dehors les jambes et les pieds. Il reste dans le noir, on ne le voit pas, il écoute ${ }^{9}$. Le lecteur est devant un texte fictif, où la convention de narration apparaît (il + focalisation interne dans « il écoute »). Trois pages plus loin, le même moment est décrit par le narrateur, cette fois à la première personne : «Je suis devant les barreaux rectangulaires de fer noir et j'attends. La fenêtre est ouverte et les cris ont cessé ». Cette fois-ci, l'utilisation de la première personne, du présent, et de l'indication corporelle visible, dans "je suis devant les barreaux », entraîne une projection de la part du lecteur dans le personnage narrateur de ce second extrait. Il ne s'agit ni d'une convention de récit fictif hétérodiégétique, ni d'une feintise de dialogue (on n'indiquerait pas à son interlocuteur où l'on se trouve), mais d'une imitation de la sensation intuitive, qu'a chacun, de son corps et de l'endroit où il se trouve (ici, le fait d'être devant les barreaux).

Ainsi, l'expérience d'une intimité de soi-même, expérience de perception de son corps (liée à des états comme percevoir, être assis, debout...), semble unanimement partagée par tous et rattachée par chacun à la prononciation/lecture du je.

Cependant, le lecteur s'identifie plus difficilement dès que l'action du je est au passé ou que l'action décrite est, dans sa particularité, ni une simple perception, ni un état visible du corps, mais une action du personnage qui ne recoupe pas une expérience que le lecteur aurait faite (par exemple, page 106 de Prison: " quand j'ai tapé ça lui a cassé le nez » ne permet pas forcément de se projeter dans ce je

${ }^{9}$ François BON, Prison, Verdier, 1999, p. 102. 
engagé dans une particularité de l'action telle que tout lecteur n'a pas forcément eu la même expérience)

On voit que l'utilisation d'une voix narrative à la première personne permet une actualisation variable : partage d'une situation de perception ou d'une action corporelle simple, par identification du lecteur au narrateur (comme s'il était cette première personne), ou bien simple identification à une situation d'auditeur.

\section{Conclusion}

Il nous est apparu que tout énonciation à la première personne induisait non seulement une identification à une situation d'auditeur, mais aussi une identification dans un je percevant (perception interne de soi-même, comme on a essayé d'en construire l'hypothèse ici, ou perception externe, comme imitation d'une réelle perception visuelle et auditive, ainsi que chez Christian Metz le dit du spectateur de cinéma). A suivre ces deux propositions, on voit qu'existe même dans L'Etranger une implication minimale du lecteur : là où nous ne nous identifions ni à la psychologie (elle est absente), ni aux actions (un meurtre n'est pas une expérience partagée par tous !), nous nous identifions au moins à la voix et au point de vue, dans le cas d'une énonciation de perceptions internes ou externes par le personnage.

Et si l'on fait l'hypothèse la plus forte, celle d'une perception interne et externe comme lieu de reconnaissance, toute lecture du je, ce $j e$ fût-il minimal, induit une identification à un je écoutant et à un je percevant. L'apparition d'une voix en je dans la narration signale l'identification du lecteur à un sujet, si lacunaire cette définition du sujet soitelle, puisqu'elle le réduit ici, mais c'est déjà beaucoup, à être un sujet de perception.

Deux conclusions peuvent être tirées de ces remarques. La première est que partager le je dans la voix narrative, au cours de la lecture d'un texte homodiégétique en première personne, c'est aussi partager la vision, quand il est question de perception: dans ce cas, voix et focalisation narratives sont confondues dans un mode narratif que le lecteur investit directement. La deuxième est plus générale : dans un texte recourant à un narrateur/personnage fantomatique à la 
première personne, la lecture induit le recours à une identification partielle, non à un sujet total (parlant, percevant, ayant des affects, pensant, agissant...), mais pourtant à une forme de sujet mis en texte, certes partiel et lacunaire : celui de l'énonciation et de la perception. Le lecteur devient le support du sujet du récit. C'est pourquoi, même s'il se donne comme fragmentaire, le sujet fait retour, pour le lecteur, selon un processus peut-être antique, celui de l'identification partielle à une instance énonçante. 